\title{
Pacific
}

Journal of

Mathematics

COMPLETE OPEN MANIFOLDS OF NON-NEGATIVE RADIAL CURVATURE

\author{
YOSHIROH MACHIGASHIRA
}




\title{
COMPLETE OPEN MANIFOLDS OF NON-NEGATIVE RADIAL CURVATURE
}

\section{YOSHIROH MACHIGASHIRA}

\begin{abstract}
We generalize the Toponogov hinge theorem and the Alexandrov convexity to the context of radial curvature, and study complete open Riemannian manifolds of non-negative radial curvature.
\end{abstract}

0. Introduction. It is well-known that a non-negative curved manifold has some interesting characters as exemplified in the Soul theorem ([CG]) or the Toponogov splitting theorem ([T]). In such theorems, Toponogov's comparison theorem plays an essential role.

Throughout this paper let $M$ be a connected complete Riemannian manifold of dimension $n \geq 2$. For a point $o \in M$, the sectional curvature $K_{M}$ of $M$ restricted to those planes that are tangent to some minimal geodesic starting from $o$ is called minimal radial curvature from $O$ and is denoted by $K_{o}^{\mathrm{min}}$. The notion of radial curvature was initiated by Klingenberg in [K] to prove a homotopy sphere theorem for compact simply-connected manifolds with $\frac{1}{4}$-pinched radial curvature. Also in the case where $M$ is noncompact and $o$ is a pole of $M$, Greene and $\mathrm{Wu}$ have shown some results related to the radial curvature from $o$ (see $[\mathbf{G W}]$ ).

In [M], it is shown that Toponogov's comparison theorem holds for the edge angles at $x_{1}$ and $x_{2}$ of a minimal geodesic triangle with vertices at $o, x_{1}$, and $x_{2}$ under suitable condition on $K_{o}^{\mathrm{min}}$. Moreover by using this fact, some results related to the radial curvature from $O$ were obtained in [M] or [MS]. For example,

Theorem 0.1 (Theorem A in [MS]). A complete noncompact Riemannian manifold $M$ which contains a point $o$ such that $K_{o}^{\min }>0$ has exactly one end.

THEOREM 0.2 (Theorem $\mathrm{C}$ in [MS]). Let $M$ be noncompact with a point $o$ such that $K_{o}^{\min } \geq 0$. If

$$
\lim _{r \rightarrow \infty} \frac{\operatorname{vol} B(o, r)}{b_{0}(r)}>\frac{1}{2}
$$


then $M$ is diffeomorphic to $\mathbf{R}^{n}$, where $\operatorname{vol} B(o, r)$ is the volume of the $r$-ball $B(o, r)$ in $M$ around $o$ and $b_{0}(r)$ is the volume of the $r$-ball of $\mathbf{R}^{n}$.

In this paper we prove that Toponogov's comparison theorem also holds for edge angles at $o$ (see Theorem 1.3) and investigate the topology of complete open manifolds of non-negative radial curvature. By using Theorem 1.3 we will obtain the

MaIn Theorem. Let $M$ be noncompact. Assume that $K_{o}^{\min } \geq 0$ for some point $o \in M$. Then:

(A) The set of critical points of the distance function from $o$ is bounded and consequently $M$ is finitely-connected.

(B) $M$ has at most two ends.

(C) If $M$ has a line, then $M$ is diffeomorphic to $N \times \mathbf{R}$, where $N$ is a hypersurface in $M$. Moreover the projection $M \rightarrow \mathbf{R}$ is a Riemannian submersion.

1. The Toponogov hinge theorem for radial curvature. For any $\delta \in$ $\mathbf{R}$, let $M^{\delta}$ denote the simply-connected surface of constant Gauss curvature $\delta$. First of all we recall the

THEOREM 1.1 (Proposition 1.1 in [M] or Theorem 1.1 in [MS]). Assume that $K_{o}^{\mathrm{min}} \geq \delta$ for $o \in M$ and $\delta \in \mathbf{R}$. Let $\gamma_{1}$ and $\gamma_{2}$ be lengthminimizing segments in $M$ with $\gamma_{1}(0)=\gamma_{2}(1)=o$ and let $\gamma_{0}$ be a length-minimizing segment such that $\gamma_{0}(0)=\gamma_{1}(1)$ and $\gamma_{0}(1)=\gamma_{2}(0)$. Then, there exist length-minimizing segments $\tilde{\gamma}_{1}, \tilde{\gamma}_{2}$, and $\tilde{\gamma}_{0}$ in $M^{\delta}$ with $\tilde{\gamma}_{1}(0)=\tilde{\gamma}_{2}(1), \tilde{\gamma}_{0}(0)=\tilde{\gamma}_{1}(1)$, and $\tilde{\gamma}_{0}(1)=\tilde{\gamma}_{2}(0)$ which are such that

$$
L\left(\gamma_{i}\right)=L\left(\tilde{\gamma}_{i}\right) \quad \text { for } i=0,1,2
$$

and

$$
\begin{aligned}
& \theta_{1}:=\angle\left(-\dot{\gamma}_{1}(1), \dot{\gamma}_{0}(0)\right) \geq \angle\left(-\dot{\tilde{\gamma}}_{1}(1), \dot{\tilde{\gamma}}_{0}(0)\right)=: \tilde{\theta}_{1}, \\
& \theta_{2}:=\angle\left(-\dot{\gamma}_{0}(1), \dot{\gamma}_{2}(0)\right) \geq \angle\left(-\dot{\tilde{\gamma}}_{0}(1), \dot{\tilde{\gamma}}_{2}(0)\right)=: \tilde{\theta}_{2} .
\end{aligned}
$$

Moreover if $\theta_{1}=\tilde{\theta}_{1} \neq \pi$, then there exists a piece of totally geodesic surface of constant curvature $\delta$ bounded by $\gamma_{1}, \gamma_{0}$, and a minimizing geodesic joining o to $\gamma_{0}(1)$ (which is not necessarily $\gamma_{2}$ ) in $M$.

The above theorem is shown by dividing $\gamma_{0}$ into sufficiently small sub-arcs $\left\{\left.\gamma_{0}\right|_{\left[t_{t-1}, t_{i}\right]}\right\}_{i=1, \ldots, N}$, where $t_{0}=0$ and $t_{N}=1$, and applying Berger's comparison theorem to obtain the angle estimates at 
$\gamma_{0}\left(t_{i-1}\right)$ and $\gamma_{0}\left(t_{i}\right)$ of a geodesic triangle $\Delta\left(o, \gamma_{0}\left(t_{i-1}\right), \gamma_{0}\left(t_{i}\right)\right.$ ) (cf. Theorem 2.2 in [CE]). If $\theta_{1}=\tilde{\theta}_{1} \neq \pi$, then the angles at $\gamma_{0}\left(t_{i-1}\right)$ and $\gamma_{0}\left(t_{i}\right)$ of any geodesic triangle $\Delta\left(o, \gamma_{0}\left(t_{i-1}\right), \gamma_{0}\left(t_{i}\right)\right)$ must be equal to the angles of the corresponding triangle in $M^{\delta}$ respectively for $i=1, \cdots, N-1$. Also the angle at $\gamma_{0}\left(t_{N-1}\right)$ of $\Delta\left(o, \gamma_{0}\left(t_{N-1}\right), \gamma_{2}(0)\right)$ equals the angle of the corresponding triangle. Moreover for every $i=1, \cdots, N$, there is a minimal geodesic $\gamma$ joining $o$ to $\gamma_{0}\left(t_{i}\right)$ such that $\gamma \subset \exp _{o}(X)$, where $X \subset T_{o} M$ is the plane spanned by $\dot{\gamma}_{1}(0)$ and the vector parallel to $\dot{\gamma}_{0}(0)$ along $\gamma_{1}$. Hence we obtain the minimal geodesic $\gamma_{2}^{\prime}$ joining $o$ to $\gamma_{0}(1)=\gamma_{2}(0)$ such that $\dot{\gamma}_{2}^{\prime}(0) \subset \exp _{o}(X)$ and a totally geodesic surface of constant curvature $\delta$ bounded by $\gamma_{1}, \gamma_{2}^{\prime}$ and $\gamma_{0}$. (For detail see $[\mathbf{M}]$.)

We can check the following corollary by dividing a geodesic triangle into two triangles.

COROLLARY 1.2. Under the assumption of Theorem 1.1,

$$
d_{M}\left(o, \gamma_{0}(s)\right) \geq d_{\delta}\left(\tilde{o}, \tilde{\gamma}_{0}(s)\right) \quad \text { for } s \in[0,1],
$$

where $d_{M}$ and $d_{\delta}$ denote the distance functions on $M$ and $M^{\delta}$ respectively.

The following theorem implies that edge angles at $o$ can be compared.

Theorem 1.3. Assume that $K_{o}^{\text {min }} \geq \delta$ for $o \in M$ and $\delta \in \mathbf{R}$. For any minimizing geodesics $\sigma_{1}:[0,1] \rightarrow M$ and $\sigma_{2}:[0,1] \rightarrow M$ starting from $O$, we have the following results

(1) Let $\tilde{\sigma}_{i}:[0,1] \rightarrow M^{\delta}$ for $i=1,2$ be minimizing geodesics starting from same point such that

$$
L\left(\sigma_{i}\right)=L\left(\tilde{\sigma}_{i}\right) \quad \text { for } i=0,1,2
$$

and

$$
\angle\left(\dot{\sigma}_{1}(0), \dot{\sigma}_{2}(0)\right)=\angle\left(\dot{\tilde{\sigma}}_{1}(0), \dot{\tilde{\sigma}}_{2}(0)\right) .
$$

Then

$$
d_{M}\left(\sigma_{1}(1), \sigma_{2}(1)\right) \leq d_{\delta}\left(\tilde{\sigma}_{1}(1), \tilde{\sigma}_{2}(1)\right) .
$$

(2) (The Alexandrov convexity). Let $\tilde{\theta}_{s, t}$ be the angle at of the triangle $\tilde{\Delta}\left(\tilde{o}, \tilde{x}_{s}, \tilde{y}_{t}\right)$ in $M^{\delta}$ corresponding to $\Delta\left(o, \sigma_{1}(s), \sigma_{2}(t)\right)$ in $M$. Then $\tilde{\theta}_{s, t}$ is monotone non-increasing in $s, t$.

(3) In (1), if equality holds, then there is a piece of totally geodesic surface of constant curvature $\delta$ bounded by $\sigma_{1}, \sigma_{2}$ and a minimal geodesic joining $\sigma_{1}(1)$ to $\sigma_{2}(1)$. 
Proof of Theorem 1.3. (1) We work with $M^{\delta-\epsilon}$ instead of $M^{\delta}$, where $\epsilon$ is any small positive number.

Put

$$
t_{0}:=\sup _{t \in[0,1]}\left\{t \mid \text { for } s \leq t, d_{M}\left(\sigma_{1}(s), \sigma_{2}(s)\right) \leq d_{\delta-\epsilon}\left(\tilde{\sigma}_{1}(s), \tilde{\sigma}_{2}(s)\right)\right\} .
$$

Then Rauch's comparison theorem implies that $t_{0}>0$. Suppose that $t_{0}<1$. Then we see that

$$
d_{M}\left(\sigma_{1}\left(t_{0}\right), \sigma_{2}\left(t_{0}\right)\right)=d_{\delta-\epsilon}\left(\tilde{\sigma}_{1}\left(t_{0}\right), \tilde{\sigma}_{2}\left(t_{0}\right)\right) .
$$

Thus we can apply Theorem 1.1 to the geodesic triangles

$$
\Delta\left(o, \sigma_{1}\left(t_{0}\right), \sigma_{2}\left(t_{0}\right)\right) \quad \text { in } M
$$

and

$$
\tilde{\Delta}\left(\tilde{\sigma}_{1}(0), \tilde{\sigma}_{1}\left(t_{0}\right), \tilde{\sigma}_{2}\left(t_{0}\right)\right) \text { in } M^{\delta-\epsilon},
$$

that is, if we let $\theta_{i}$ and $\tilde{\theta}_{i}$ for $i=1,2$ be the angles at $\sigma_{i}\left(t_{0}\right)$ and $\tilde{\sigma}_{i}\left(t_{o}\right)$, then

$$
\theta_{1} \geq \tilde{\theta}_{1} \text { and } \theta_{2} \geq \tilde{\theta}_{2} .
$$

In the case where $\theta_{1}>\tilde{\theta}_{1}$, the first variation formula implies that

$$
d_{M}\left(\sigma_{1}\left(t_{0}+h\right), \sigma_{2}\left(t_{0}+h\right)\right)<d_{\delta-\epsilon}\left(\tilde{\sigma}_{1}\left(t_{0}+h\right), \tilde{\sigma}_{2}\left(t_{0}+h\right)\right)
$$

for sufficiently small $h>0$. This contradicts the definition of $t_{0}$. Next we consider the case where $\theta_{1}=\tilde{\theta}_{1}$. Since the assumption that $t_{0}<1$ says $\theta_{1} \neq \pi$, the later half of Theorem 1.1 implies that there exists a piece of totally geodesic surface of constant curvature $\delta-\epsilon$ bounded by $\left.\sigma_{1}\right|_{\left[0, t_{0}\right]},\left.\sigma_{2}\right|_{\left[0, t_{0}\right]}$ and a minimal geodesic joining $\sigma_{1}\left(t_{0}\right)$ to $\sigma_{2}\left(t_{0}\right)$. This contradicts $K_{o}^{\mathrm{min}} \geq \delta$. This completes the proof of (1).

(2) It suffice to show that for arbitrary fixed $s \in(0,1]$ and $t \in$ $(0,1)$,

$$
\tilde{\theta}_{s, t} \geq \tilde{\theta}_{s, t+h} \text { for small } h>0 .
$$

By continuty of $\tilde{\theta}_{s, t}$, we may assume $s<1$. Put

$$
\theta:=\angle\left(\dot{\sigma}_{1}(0), \dot{\sigma}_{2}(0)\right) \text {. }
$$

Restating (1), we see that $\tilde{\theta}_{s, t} \leq \theta$ for all $s, t \in[0,1]$. Thus in the case where $\tilde{\theta}_{s, t}=\theta$, clearly (1.1) holds. Hence we consider only the case $\tilde{\theta}_{s, t}<\theta$.

Let $\tilde{\sigma}$ be minimal geodesic in $M^{\delta}$ starting from $\tilde{o}$ and passing $y_{t}$ parameterized as $\tilde{\sigma}(t)=\tilde{y}_{t}$. From Theorem 1.1, the angle at $\tilde{y}_{t}$ of 
$\tilde{\Delta}\left(\tilde{o}, \tilde{x}_{s}, \tilde{y}_{t}\right)$ does not exceed the angle at $\sigma_{2}(t)$ of $\Delta\left(o, \sigma_{1}(s), \sigma_{2}(t)\right)$. If the angles are equal to each other, by the latter half of Theorem 1.1 , it must be that $\tilde{\theta}_{s, t} \geq \theta$, because the minimal geodesic joining $o$ to $\sigma_{1}(s)$ is unique. This contradicts $\tilde{\theta}_{s, t}<\theta$. If the angle at $y_{t}$ is smaller than the angle at $\sigma_{2}(t)$, then the first variation formula implies that

$$
d_{\delta}(\tilde{o}, \tilde{\sigma}(t+h)) \geq d_{M}\left(o, \sigma_{2}(t+h)\right)
$$

for small $h>0$, that is,

$$
\tilde{\theta}_{s, t+h} \leq \tilde{\theta}_{s, t} .
$$

This completes the proof of (2).

(3) We apply (2) to obtain that if

$$
d_{M}\left(\sigma_{1}(1), \sigma_{2}(1)\right)=d_{\delta}\left(\tilde{\sigma}_{1}(1), \tilde{\sigma}_{2}(1)\right),
$$

then $\theta=\tilde{\theta}_{s, t}$ for all $s, t \in[0,1]$. Hence the angles at $\tilde{x}_{s}$ and $\tilde{y}_{t}$ of $\tilde{\Delta}\left(\tilde{o}, \tilde{x}_{s}, \tilde{y}_{t}\right)$ equal the angles at $\sigma_{1}(s)$ and $\sigma_{2}(t)$ of $\Delta\left(o, \sigma_{1}(s), \sigma_{2}(t)\right)$ respectively for all $s, t \in(0,1)$. Thus there is a piece of totally geodesic surface of constant curvature $\delta$ bounded by $\left.\sigma_{1}\right|_{[0, s]},\left.\sigma_{2}\right|_{[0, t]}$ and a minimal geodesic $\sigma_{s, t}$ joining $\sigma_{1}(s)$ to $\sigma_{2}(t)$. Hence $\dot{\sigma}_{s, t}(0)$ is contained the plane spanned by $\dot{\sigma}_{1}(s)$ and the vector parallel to $\dot{\sigma}_{2}(0)$ along $\left.\sigma_{1}\right|_{[0, s]}$. Taking $s, t \rightarrow 1$, we obtain a minimal geodesic joining $\sigma_{1}(1)$ to $\sigma_{2}(1)$.

The proof of Theorem 1.3 is completed.

REMARK 1.4. By using Theorem 1.3 (2), we can construct the ideal boundary $M(\infty)$ of a complete open manifold $M$ of nonnegative radial curvature and the Titz metric on it. However it is not needed in this article.

2. Proof of the main theorem. Now part (A) of the main theorem is shown directly from Theorem 1.3 in the same way as the proof of the corollary to Theorem 1.5.A in [G] or Corollary 2.9 in [C].

In the remainder of this paper, we agree that geodesics will be parameterized by the arc-length.

Proof of part (B) of the main theorem. Suppose that $M$ has three or more ends. Then there are three rays $\gamma_{1}, \gamma_{2}$, and $\gamma_{3}$ starting from $o$ going to different ends. Let $l_{t}$ be a minimal geodesic joining $\gamma_{1}(t)$ to $\gamma_{2}(t)$ and $\tilde{\theta}_{t}$ the angle at $\tilde{o} \in \mathbf{R}^{2}$ of $\tilde{\Delta}\left(\tilde{o}, \tilde{x}_{t}, \tilde{y}_{t}\right)$ in $\mathbf{R}^{2}$ such that $d_{0}\left(\tilde{o}, \tilde{x}_{t}\right)=d_{0}\left(\tilde{o}, \tilde{y}_{t}\right)=t$ and $d_{0}\left(\tilde{x}_{t}, \tilde{y}_{t}\right)=L\left(l_{t}\right)$. Since the distance between $o$ and $l_{t}$ is bounded from above by some constant 
$C$ independent of $t$, Corollary 1.2 implies that the distance between $\tilde{o}$ and the segment joining $\tilde{x}_{t}$ to $\tilde{y}_{t}$ is also bounded by $C$. Thus we see that

$$
\tilde{\theta}_{t} \rightarrow \pi \quad \text { as } t \rightarrow \infty
$$

and consequently

$$
\angle\left(\dot{\gamma}_{1}(0), \dot{\gamma}_{2}(0)\right)=\pi
$$

Similarly, we have that

$$
\angle\left(\dot{\gamma}_{2}(0), \dot{\gamma}_{3}(0)\right)=\angle\left(\dot{\gamma}_{3}(0), \dot{\gamma}_{1}(0)\right)=\pi \text {. }
$$

This contradicts and hence completes the proof of (B).

In Lemma 1.3 in [MS] it is shown that if a non-negative minimal radial curved $M$ with base point $o$ has a line, then there is a line passing through $o$. We will show that a similar thing is realized for any $x \in M$. We define a Busemann function $F_{\gamma}$ on noncompact $M$ for a ray $\gamma$ by

$$
F_{\gamma}(x):=\lim _{t \rightarrow \infty}\left[t-d_{M}(x, \gamma(t))\right] \quad \text { for } x \in M .
$$

LEMMA 2.1. Under the assumption of the main theorem, if there is a line $\sigma$ through $o$, then for any $x \in M$ there is a unique line $l_{x}$ through $x$ which is biasymptotic to $\sigma$. Moreover there exists a flat totally geodesic strip bounded by $\sigma(\mathbf{R})$ and $l_{x}(\mathbf{R})$.

Proof. Choose the parameter of $\sigma$ such that $\sigma(0)=o$ and set $\sigma_{ \pm}(t):=\sigma( \pm t)$ for $t \geq 0$. Let $\beta$ be a minimal geodesic joining $o$ to $x$ and put $\theta_{ \pm}:=\angle\left(\dot{\beta}(0), \dot{\sigma}_{ \pm}(0)\right)$. Let $\tilde{\theta}_{ \pm t}$ be the angles at $\tilde{o} \in \mathbf{R}^{2}$ of $\tilde{\Delta}\left(\tilde{o}, \tilde{x}, \tilde{y}_{ \pm t}\right)$ in $\mathbf{R}^{2}$ corresponing to $\Delta\left(o, x, \sigma_{ \pm}(t)\right)$ in $M$, and put $\tilde{\theta}_{ \pm \infty}:=\lim _{t \rightarrow \infty} \tilde{\theta}_{ \pm t}$. (Theorem 1.3 (2) guarantees the existence of $\tilde{\theta}_{ \pm \infty}$.) Then

$$
F_{\sigma_{ \pm}}(x)=d(o, x) \cos \tilde{\theta}_{ \pm \infty}
$$

Thus we obtain that

$$
\cos \tilde{\theta}_{+\infty}+\cos \tilde{\theta}_{-\infty} \leq 0
$$

because it follows from the triangle inequality that

$$
F_{\sigma_{+}}(x)+F_{\sigma_{-}}(x) \leq 0 .
$$

On the other hand, by Theorem 1.3 we see that

$$
\tilde{\theta}_{+\infty}+\tilde{\theta}_{-\infty} \leq \theta_{+}+\theta_{-}=\pi \text {. }
$$


The formulas (2.1) and (2.2) imply that $\tilde{\theta}_{+\infty}+\tilde{\theta}_{-\infty}=\pi$, and consequently $\tilde{\theta}_{+t}+\tilde{\theta}_{-t}=\pi$ for all $t>0$ from Theorem 1.3. Thus we obtain that $\tilde{\theta}_{ \pm t}=\theta_{ \pm}$for all $t>0$ and there are two pieces of totally geodesic surface of constant curvature 0 bounded by $\left.\sigma_{ \pm}\right|_{[0, t]}, \beta$ and $l_{ \pm t}$, where $l_{ \pm t}$ are geodesics joining $x$ to $\sigma_{ \pm}(t)$. Hence there exist two rays $l_{x \pm}$ starting from $x$ and asymptotic to $\sigma_{ \pm}$such that $\dot{l}_{x \pm}(0)$ are parallel to $\dot{\sigma}_{ \pm}(0)$ along $\beta$. Moreover there exists a flat totally geodesic strip bounded by $\sigma$ and $l_{x}:=l_{x+} \cup l_{x_{-}}$. To prove that $l_{x}$ is a line, we consider $l_{x+}(-s)=: x_{s}$ for arbitrary $s>0$ instead of $x$. Then $\left.l_{x-}\right|_{[s, \infty]}$ is the unique ray starting from $x_{s}$ and asymptotic to $\sigma_{-}$because $x_{s}$ is an interior point of a ray $l_{x-}$ (see Theorem 1.1 in [S]). Hence we see that $\left.l_{x+}\right|_{[-s, \infty]}$ is a ray and $l_{x}$ must be a line. This completes the proof of Lemma 2.1 .

Proof of part (C) of the main theorem. Let $\sigma$ be a line passing through $o$ parameterized as $\sigma(0)=o$, constructed in Lemma 1.3 in [MS]. Let $\sigma_{+}$be $\left.\sigma\right|_{[0, \infty)}$ and put $N_{s}:=\left(F_{\sigma_{+}}\right)^{-1}(s)$ for $s \in \mathbf{R}$. Then $N_{s}$ is a smooth hypersuface of $M$ because the gradient vector at $x$ of $F_{\sigma_{+}}$is unique and its length equals 1 for any $x \in M$ by Lemma 2.1. For $x \in M$ let $x_{0} \in l_{x}(\mathbf{R})$ be the point such that $d_{M}\left(o, x_{0}\right)=$ $d_{M}\left(o, l_{x}(\mathbf{R})\right)$, where $l_{x}$ is as in Lemma 3.1. Then Lemma 3.1 implies that $x_{0}$ is unique and contained in $N_{0}$. Thus we can define a map $g_{s}: N_{s} \rightarrow N_{0}$ for all $s \in \mathbf{R}$ by $g_{s}(x):=x_{0}$. This map is clearly bijective and a local diffeomorphism, that is, a global diffeomorphism. Hence at last we obtain the desired map $G: M \rightarrow N_{0} \times \mathbf{R}$ by $G(x):=$ $\left(x_{0}, F_{\sigma_{+}}(x)\right)$.

REMARK 2.2. Each hypersurface $N_{s}$ is a star-shaped subset of $M$, that is, a minimal geodesic joining $\sigma(s)$ to a point in $N_{s}$ is contained in $N_{s}$. Moreover if the Busemann functions $F_{\left.\sigma\right|_{[a, \infty]}}$ and $F_{\left.\sigma\right|_{[-\infty, a]}}$ for $a \in \mathbf{R}$ are convex, then $N_{s}$ is totally convex for any $s \in \mathbf{R}$ and the map $G$ is an isometry. But we do not know whether it is true or not that the Busemann function for a ray passing through $O$ is convex under the condition $K_{o}^{\min } \geq 0$.

Acknowledgment. The author would like to express thanks to Professor Katsuhiro Shiohama for his advice and encouragement. The author also thanks Takashi Shioya and Takao Yamaguchi. Remark 1.4 is Shioya's idea. He is preparing a paper related to the ideal boundary of a nonnegative curved manifold and the Titz metric on it. 


\section{REFERENCES}

[C] J. Cheeger, Critical points of distance functions and applications to geometry, Proc. Sympos. Pure Math., (to appear).

[CG] J. Cheeger and D. Gromoll, On the structure of complete manifolds and nonnegative curvature, Ann. of Math., 96 (1972), 413-443.

[G] M. Gromov, Curvature, diameter and Betti numbers, Comment. Math. Helv., 56 (1981), 179-195.

[GW] R. E. Greene and H. Wu, Function Theory on Manifolds Which Possess a Pole, Lecture Notes in Math., vol. 699, Springer-Verlag, Berlin, Heidelberg, New York, 1979.

[K] W. Klingenberg, Manifolds with restricted conjugate locus, Ann. of Math., 78 (1963), 527-547.

[M] Y. Machigashira, Manifolds with pinched radial curvature, Proc. Amer. Math. Soc., 118 (1993), 979-985.

[MS] Y. Machigashira and K. Shiohama, Riemannian manifolds with positive radial curvature, Japan. J. Math., (to appear).

[S] K. Shiohama, Topology of complete noncompact manifolds, Geometry of Geodesics and Related Topics, Advanced Studies in Pure Mathematics, vol. 3, 1984, pp. 423-450.

[T] V. Toponogov, Riemannian spaces with straight lines, Amer. Math. Soc. Transl. Ser. 2, 37 (1964), 287-290.

Received May 21, 1992.

DEPARTMENT OF INFORMATION SCIENCE

SAGA UNIVERSITY

SAGA 840 , JAPAN 


\section{PACIFIC JOURNAL OF MATHEMATICS}

Founded by

E. F. BECKENBACH (1906-1982) F. Wolf (1904-1989)

\section{EDITORS}

Sun-Yung A. Chang

(Managing Editor)

University of California

Los Angeles, CA 90024-1555

chang@math.ucla.edu

\section{F. Michael Christ}

University of California

Los Angeles, CA 90024-1555

christ@math.ucla.edu

Herbert Clemens

University of Utah

Salt Lake City, UT 84112

clemens@math.utah.edu

\author{
ThOMAs ENRIGHT \\ University of California, San Diego \\ La Jolla, CA 92093 \\ tenright@ucsd.edu \\ Nicholas ERcolaNI \\ University of Arizona \\ Tucson, AZ 85721 \\ ercolani@math.arizona.edu \\ R. FINN \\ Stanford University \\ Stanford, CA 94305 \\ finn@gauss.stanford.edu \\ VAUGHAN F. R. Jones \\ University of California \\ Berkeley, CA 94720 \\ vfr@math.berkeley.edu
}

\author{
STEVEn KerCKHOFF \\ Stanford University \\ Stanford, CA 94305 \\ spk@gauss.stanford.edu
}

Martin ScharlemanN

University of California

Santa Barbara, CA 93106

mgscharl@math.ucsb.edu

\author{
Harold Stark \\ University of California, San Diego \\ La Jolla, CA 92093 \\ V. S. VARADARAJAN \\ University of California \\ Los Angeles, CA 90024-1555 \\ vsv@math.ucla.edu
}

\section{SUPPORTING INSTITUTIONS}

UNIVERSITY OF ARIZONA

UNIVERSITY OF BRITISH COLUMBIA

CALIFORNIA INSTITUTE OF TECHNOLOGY

UNIVERSITY OF CALIFORNIA

UNIVERSITY OF MONTANA

UNIVERSITY OF NEVADA, RENO

NEW MEXICO STATE UNIVERSITY

OREGON STATE UNIVERSITY

\author{
UNIVERSITY OF OREGON \\ UNIVERSITY OF SOUTHERN CALIFORNIA \\ STANFORD UNIVERSITY \\ UNIVERSITY OF HAWAII \\ UNIVERSITY OF UTAH \\ WASHINGTON STATE UNIVERSITY \\ UNIVERSITY OF WASHINGTON
}

The Supporting Institutions listed above contribute to the cost of publication of this Journal, but they are not owners or publishers and have no responsibility for its content or policies.

Mathematical papers intended for publication in the Pacific Journal of Mathematics should be in typed form or offset-reproduced (not dittoed), double spaced with large margins. Please do not use built up fractions in the text of the manuscript. However, you may use them in the displayed equations. Underline Greek letters in red, German in green, and script in blue. The first paragraph must be capable of being used separately as a synopsis of the entire paper. In particular it should contain no bibliographic references. Please propose a heading for the odd numbered pages of less than 35 characters. Manuscripts, in triplicate, may be sent to any one of the editors. Please classify according to the 1991 Mathematics Subject Classification scheme which can be found in the December index volumes of Mathematical Reviews. Supply name and address of author to whom proofs should be sent. All other communications should be addressed to the managing editor, or Julie Honig, University of California, Los Angeles, California 90024-1555.

There are page-charges associated with articles appearing in the Pacific Journal of Mathematics. These charges are expected to be paid by the author's University, Government Agency or Company. If the author or authors do not have access to such Institutional support these charges are waived. Single authors will receive 75 free reprints; joint authors will receive a total of 100 free reprints. Additional copies may be obtained at cost in multiples of 50 .

The Pacific Journal of Mathematics (ISSN 0030-8730) is published monthly except for July and August. Regular subscription rate: $\$ 215.00$ a year (10 issues). Special rate: $\$ 108.00$ a year to individual members of supporting institutions.

Subscriptions, orders for numbers issued in the last three calendar years, and changes of address should be sent to Pacific Journal of Mathematics, P.O. Box 4163, Berkeley, CA 94704-0163, U.S.A. Old back numbers obtainable from Kraus Periodicals Co., Route 100, Millwood, NY 10546.

The Pacific Journal of Mathematics at University of California, c/o Department of Mathematics, 981 Evans Hall, Berkeley, CA 94720 (ISSN 0030-8730) is published monthly except for July and August. Second-class postage paid at Berkeley, CA 94704, and additional mailing offices. POSTMASTER: send address changes to Pacific Journal of Mathematics, P.O. Box 4163, Berkeley, CA 94704-0163.

PUBLISHED BY PACIFIC JOURNAL OF MATHEMATICS at University of California, Berkeley, CA 94720, A NON-PROFIT CORPORATION

This publication was typeset using $\mathcal{A} \mathcal{M} \mathcal{S}-\mathrm{T}_{\mathrm{E}} \mathrm{X}$,

the American Mathematical Society's $T_{E} X$ macro system.

Copyright (c) 1994 by Pacific Journal of Mathematics 


\section{PACIFIC JOURNAL OF MATHEMATICS}

Volume $165 \quad$ No. $1 \quad$ September 1994

The effect of dimension on certain geometric problems of irregularities of 1 distribution

RALPH ALEXANDER

The structure of $\mathrm{sl}(2,1)$-supersymmetry: irreducible representations and primitive ideals

Didier ARnAL, HÉdi BENAMOR and GEORges PinCZON

Periods and Lefschetz zeta functions

Josefina CASASAYas, JaUme Llibre and ANA Nunes

On closed hypersurfaces of constant scalar curvatures and mean curvatures in 67 $S^{n+1}$

\section{SHAOPING CHANG}

$R$-groups and elliptic representations for $\mathrm{SL}_{n}$

DAVID GOLDBERG

The boundary distortion of a quasiconformal mapping

JuHA HeINONEN and PEKKA KosKela

Strongly approximately transitive group actions, the Choquet-Deny theorem, 115 and polynomial growth

\section{WOJCIECH JAWORSKI}

$q$-canonical commutation relations and stability of the Cuntz algebra

PALle E. T. Jorgensen, L. M. Schmitt and Reinhard Frank

WERNER

Complete open manifolds of non-negative radial curvature

YOSHIROH MACHIGASHIRA

Perturbations of certain reflexive algebras

DAVID RYDER PITTS

Interpolation submanifolds of the unitary group 